\title{
Mitigation of Influenza B Epidemic with School Closures, Hong Kong, 2018
}

\section{Sheikh Taslim Ali, Benjamin J. Cowling, Eric H.Y. Lau, Vicky J. Fang, Gabriel M. Leung}

In winter 2018, schools in Hong Kong were closed 1 week before the scheduled Chinese New Year holiday to mitigate an influenza B virus epidemic. The intervention occurred after the epidemic peak and reduced overall incidence by $\approx 4.2 \%$. School-based vaccination programs should be implemented to more effectively reduce influenza illnesses.

$\mathrm{H}$ ong Kong, China, located on the coast south of Guangdong Province, has a subtropical climate and a population of 7.3 million. In Hong Kong, influenza epidemics occur during winter every year and sometimes during other seasons (1). One of the interventions that has been used by Hong Kong health authorities to control influenza epidemics is school closures; this intervention was previously applied in 2008 (2) and 2009 (3). During winter 2017-18, an epidemic of influenza B, Yamagata lineage, occurred in Hong Kong. The local media focused on this epidemic for 3 reasons. First, the occurrence of severe influenza cases in Hong Kong (4) attracted public concern. Second, the number of school outbreaks reported to the Centre for Health Protection in Hong Kong far exceeded the number reported in previous years (4). Third, a severe epidemic of influenza $\mathrm{A}(\mathrm{H} 3 \mathrm{~N} 2)$ was ongoing in the United States (5), which further increased local concern about influenza in general.

On February 7, 2018, the Hong Kong government announced that all 1,600 kindergartens, primary schools, and special needs schools in Hong Kong would close the following day, 1 week before the Chinese New Year school holiday, which in most schools was scheduled for February 15-23. Thus, in total, schools were closed for 2.5 weeks. We reviewed surveillance data on influenza and influenzalike illness (ILI) activity in Hong Kong to infer the effect of school closures on community transmission.

\section{The Study}

As in previous studies, we used ILI surveillance data to indicate the incidence of influenza virus infections in the community $(1,6,7)$. The Centre for Health Protection tracks a sentinel network of private doctors and reports the rates

Author affiliation: The University of Hong Kong, Hong Kong, China

DOI: https://doi.org/10.3201/eid2411.180612 of outpatient consultations for ILI per 1,000 patient consultations every week (4), and the Public Health Laboratory Services branch reports the proportion of respiratory specimens testing positive for influenza virus by type and subtype every week (8). We multiplied the weekly ILI rates by the weekly influenza $\mathrm{B}$ virus detection rates to obtain a proxy (hereafter ILI+ proxy) measure of the number of cases of influenza B virus infection each week (Figure, panel A). We have previously shown that this ILI+ proxy provides an estimate that correlates linearly with the incidence of hospitalizations for influenza A(H1N1)pdm09 in Hong Kong ( 6 ); some have argued that this metric is a better linear correlate of the incidence of influenza illness than ILI rates alone or laboratory detection rates alone (9).

We calculated the ILI+ proxy for influenza B to infer the rate of person-to-person transmission of influenza B virus throughout the epidemic. We used the methods proposed by Cauchemez et al. (10) to estimate transmissibility by the effective reproduction number, $\mathrm{R}$, which represents the average number of secondary infections that result from a primary case of infection at time $t$ (online Technical Appendix, https://wwwnc.cdc.gov/EID/article/24/11/180612-Techapp1.pdf). When $\mathrm{R}_{t}$ exceeds 1 , the epidemic is capable of spreading. We used flexible cubic splines to model the weekly influenza B ILI+ proxy values and interpolate daily ILI+ proxy values. We then estimated daily $\mathrm{R}_{t}$ values from the daily influenza B ILI+ proxy values (7). We considered the serial interval distribution as the Weibull distribution, with a mean of 3.2 days and SD of 1.3 days (11). The estimated $\mathrm{R}_{t}$ was 1.03 (95\% CI 0.73-1.34) before the start of the school closure and 0.87 (95\% CI 0.54-1.21) during the closure week, corresponding to a $16 \%(95 \% \mathrm{CI}$ $10 \%-26 \%$ ) reduction in transmissibility (Figure, panel B).

We then simulated the ILI+ proxy for influenza B under the counterfactual scenario of no school closures during February $8-14$. Because $\mathrm{R}_{t}$ is affected by the depletion of the susceptible population $(h$, cumulative ILI+ proxy for influenza B at time $t)$ and school closure $\left(C_{t}\right.$, indicator variable at time $t$ ), we first fitted a multivariable log-linear regression model for $\mathrm{R}_{t}$ with $h_{t}$ and $C_{t}$ as explanatory variables (online Technical Appendix). Using these estimated coefficients in a regression model, we then constructed the transmission rate $\left(\beta_{t}\right.$, function of initial transmission rate $\beta_{0}$ and $C_{t}$ ) for a susceptible-exposed-infected-recovered compartmental model to simulate incidence over time. To 

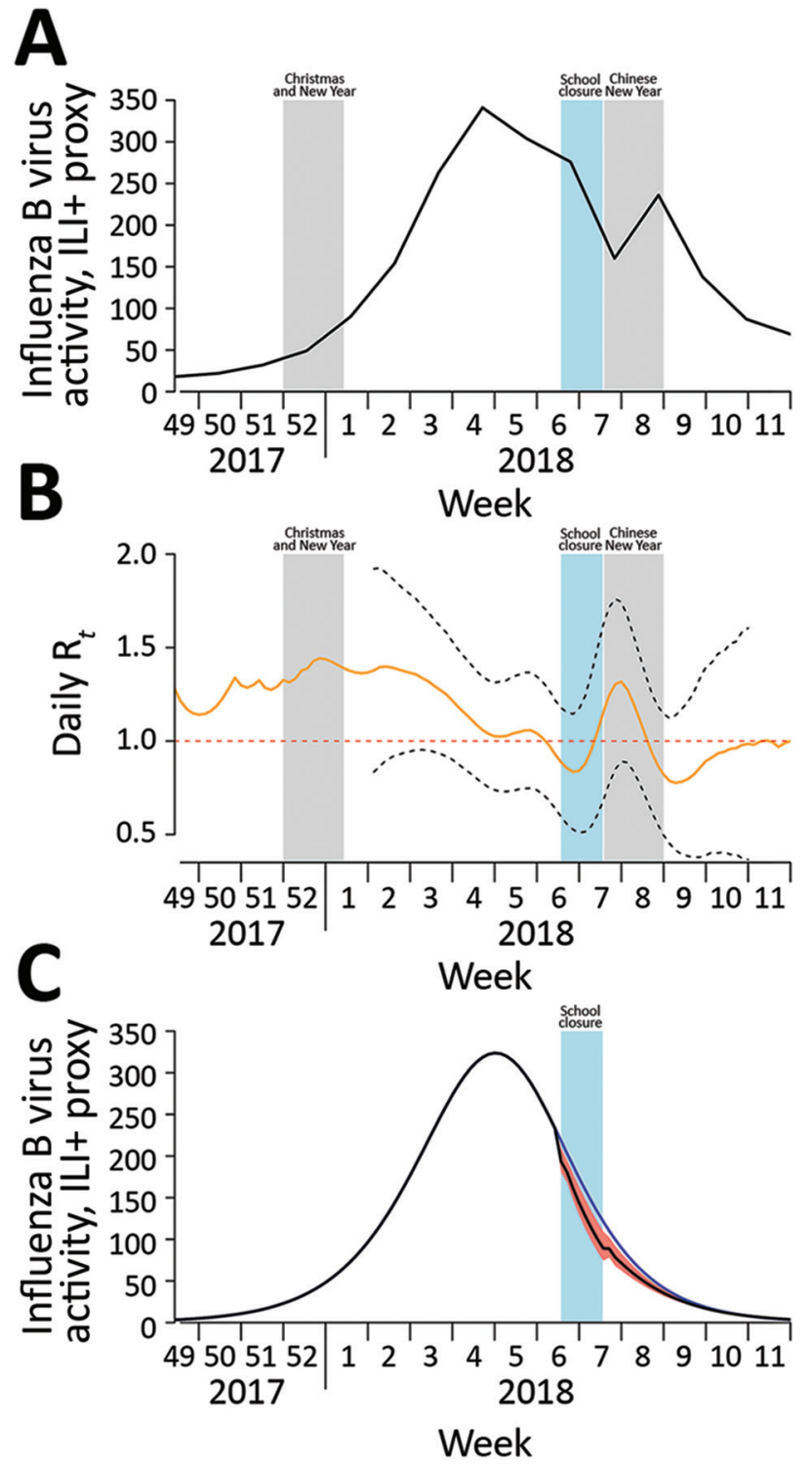

Figure. Influenza B virus activity, by epidemiologic week, Hong Kong, December 2017-March 2018. A) Incidence of influenza $B$ virus measured by using the ILI+ proxy for influenza $B$, which is calculated by multiplying the weekly rate of ILI per 1,000 consultations by the weekly proportion of respiratory specimens submitted to the Public Health Laboratory Services (Hong Kong) that tested positive for influenza B virus (online Technical Appendix Table 2, https://wwwnc.cdc.gov/EID/article/24/11/18-0612-Techapp1.pdf). Shaded bars show school closure dates. B) Daily real-time estimate of transmissibility $\left(R_{t}\right)$ of influenza $B$ virus. Black dashed lines indicate pointwise $95 \% \mathrm{Cls}$; red dashed line indicates transmission threshold. Shaded bars show school closure dates. C) Simulated incidence of influenza $B$ virus with and without implementation of school closure (shaded bar) in Hong Kong during February 8-14, 2018. Blue line indicates the number of cases occurring during the hypothetical scenario of no school closure; black line indicates the number occurring with school closure, which reduced transmissibility by $16 \%$. The difference between these 2 lines represents the $4.2 \%$ reduction in incidence of infections; red shading indicates $95 \% \mathrm{Cl}$. $\mathrm{ILI}$, influenza-like illness; $\mathrm{R}_{\mathrm{t}}$, effective reproduction number at time $t$. simulate incidence under no school closure, we set $C_{t}$ to 0 for the period February 8-14 (online Technical Appendix).

Under the simulated epidemic with no school closures, the cumulative incidence for the entire epidemic was 0.527 (95\% CI 0.472-0.574); the incidence was reduced to 0.505 (95\% CI 0.494-0.519) when simulated with closures (Figure, panel C). This difference in proportions corresponds to a $4.2 \%(95 \%$ CI $1.5 \%-6.7 \%)$ reduction in infections from school closures. In sensitivity analyses, in which different levels of preexisting immunity $(0.1 \%-30 \%)$ in the population were assumed, the estimated reduction in infections ranged from $3.3 \%$ (95\% CI $1.2 \%-5.1 \%$ ) for high $(30 \%)$ preexisting immunity to $4.1 \%(95 \%$ CI $1.5 \%-6.7 \%)$ for low $(0.1 \%)$ preexisting immunity. We also simulated the effect of school closures occurring 1 or 2 weeks earlier and estimated that infections would have been reduced by $8.6 \%$ if schools closed 1 week earlier (lasting for 2 weeks) and $13.5 \%$ if schools closed 2 weeks earlier (lasting for 3 weeks) (online Technical Appendix).

\section{Conclusions}

In early 2018, schools were closed an extra week before a holiday in Hong Kong to mitigate an influenza epidemic. Closure after the epidemic peak had a small effect on transmission; we estimated a $4.2 \%$ reduction in overall incidence of infections. By the end of the 2017-18 winter season, $\approx 400$ laboratory-confirmed influenza deaths had occurred among the local population of 7.2 million, lower than the rate in the contemporaneous influenza $\mathrm{A}(\mathrm{H} 3 \mathrm{~N} 2)$ epidemic in the United States but still a rate of moderate-tohigh impact. A reduction in incidence of infections by $4.2 \%$ might have reduced hospitalizations and deaths by a similar percentage, with the caveat that hospitalizations and deaths would probably not have been equally distributed among age groups because most infections occur in children and most deaths in older adults.

The school closures were announced $<24$ hours before they began. We presume that the school closures were disruptive to parents' schedules, potentially forcing some parents to stay home from work, and that many children stayed home during closures (12). The $16 \%$ reduction in transmission we estimated was lower than that estimated for the school closures that occurred in Hong Kong during June-July 2009 (25\% reduction) (3). In 2009, the goal was to delay community transmission and spread out disease activity peak; thus, intervention before the peak was essential. In our study, $\mathrm{R}_{t}$ appeared to increase (Figure, panel B) during the Chinese New Year, probably because of increased social interactions during holiday gatherings.

We assumed that the ILI+ proxy for influenza B was linearly correlated with the incidence of infections $(1,6,7)$. This correlation could have been affected by changes in healthcare seeking behavior that might have resulted from 
private clinic closure, which occurred for a few days during the Chinese New Year holiday. This decreased healthcare access might have shifted the estimated reduction of influenza infections upward.

Influenza vaccination is considered the best preventive measure against influenza. However, $>10$ years after introduction of the influenza vaccination subsidy scheme for children, influenza vaccination coverage is still low in Hong Kong: $\approx 10 \%$ overall and $\approx 15 \%$ in children for the 2016-17 and 2017-18 winter seasons (13). To further increase influenza vaccination coverage in children, a schoolbased vaccination program should be implemented for the upcoming 2018-19 winter season.

\section{Acknowledgments}

The authors thank Julie Au and Huiying Chua for technical assistance.

This project was supported by the Harvard Center for Communicable Disease Dynamics from the National Institute of General Medical Sciences (grant no. U54 GM088558), a commissioned grant from the Health and Medical Research Fund from the Government of the Hong Kong Special Administrative Region, and the Research Grants Council of the Hong Kong Special Administrative Region, China (project no. T11-705/14N). The funding bodies had no role in study design, data collection and analysis, preparation of the manuscript, or the decision to publish.

\section{About the Author}

Dr. Ali works as a postdoctoral fellow at the World Health Organization Collaborating Centre for Infectious Disease Epidemiology and Control, School of Public Health, The University of Hong Kong in Hong Kong, China. His research interests include mathematical and statistical epidemiology, modeling of infectious diseases, and containment and mitigation policies.

\section{References}

1. Wu P, Presanis AM, Bond HS, Lau EHY, Fang VJ, Cowling BJ. A joint analysis of influenza-associated hospitalizations and mortality in Hong Kong, 1998-2013. Sci Rep. 2017;7:929. http://dx.doi.org/10.1038/s41598-017-01021-x
2. Cowling BJ, Lau EH, Lam CL, Cheng CK, Kovar J, Chan KH, et al. Effects of school closures, 2008 winter influenza season, Hong Kong. Emerg Infect Dis. 2008;14:1660-2. http://dx.doi.org/10.3201/eid1410.080646

3. Wu JT, Cowling BJ, Lau EH, Ip DKM, Ho LM, Tsang T, et al. School closure and mitigation of pandemic (H1N1) 2009, Hong Kong. Emerg Infect Dis. 2010;16:538-41. http://dx.doi.org/10.3201/eid1603.091216

4. Centre for Health Protection. Local situation of influenza activity (as of Feb 7, 2018). Flu Express. 2018;15:1-8.

5. Budd AP, Wentworth DE, Blanton L, Elal AIA, Alabi N, Barnes J, et al. Update: influenza activity-United States, October 1, 2017February 3, 2018. MMWR Morb Mortal Wkly Rep. 2018;67: 169-79. http://dx.doi.org/10.15585/mmwr.mm6706a1

6. Wong JY, Wu P, Nishiura H, Goldstein E, Lau EH, Yang L, et al. Infection fatality risk of the pandemic A(H1N1)2009 virus in Hong Kong. Am J Epidemiol. 2013;177:834-40. http://dx.doi.org/10.1093/aje/kws314

7. Ali ST, Wu P, Cauchemez S, He D, Fang VJ, Cowling BJ, et al. Ambient ozone and influenza transmissibility in Hong Kong. Eur Respir J. 2018;51:1800369. http://dx.doi.org/ 10.1183/13993003.00369-2018

8. Centre for Health Protection. Influenza virus subtyping in 2018. 2018 [cited 2018 Apr 4]. https://www.chp.gov.hk/en/statistics/ data/10/641/643/6781.html

9. Goldstein E, Cobey S, Takahashi S, Miller JC, Lipsitch M. Predicting the epidemic sizes of influenza A/H1N1, A/H3N2, and B: a statistical method. PLoS Med. 2011;8:e1001051. http://dx.doi.org/10.1371/journal.pmed.1001051

10. Cauchemez S, Boëlle PY, Thomas G, Valleron AJ. Estimating in real time the efficacy of measures to control emerging communicable diseases. Am J Epidemiol. 2006;164:591-7. http://dx.doi.org/10.1093/aje/kwj274

11. Cowling BJ, Chan KH, Fang VJ, Lau LLH, So HC, Fung ROP, et al. Comparative epidemiology of pandemic and seasonal influenza A in households. N Engl J Med. 2010;362:2175-84. http://dx.doi.org/10.1056/NEJMoa0911530

12. Cauchemez S, Ferguson NM, Wachtel C, Tegnell A, Saour G, Duncan B, et al. Closure of schools during an influenza pandemic. Lancet Infect Dis. 2009;9:473-81. http://dx.doi.org/10.1016/ S1473-3099(09)70176-8

13. Chiu SS, Kwan MYW, Feng S, Wong JSC, Leung CW, Chan ELY, et al. Interim estimate of influenza vaccine effectiveness in hospitalised children, Hong Kong, 2017/18. Euro Surveill. 2018;23. http://dx.doi.org/10.2807/1560-7917.ES.2018.23.8.18-00062

Address for correspondence: Benjamin J. Cowling, School of Public Health, Li Ka Shing Faculty of Medicine, The University of Hong Kong, 7 Sassoon Rd, Pokfulam, Hong Kong, China; email: bcowling@hku.hk 